\title{
VENTAS INFORMALES EN EL ESPACIO PÚBLICO EN VILLAVICENCIO (COLOMBIA)*
}

\author{
Recibido: 23 de noviembre de 2016 - Aprobado: 20 de febrero de 2018 \\ DOI: $10.22395 /$ seec.v21n46a6 \\ Edisson Stiven Castro Escobar ${ }^{* *}$ \\ Duván Emilio Ramírez Ospina** \\ Héctor Mauricio Serna Gómez ${ }^{* * *}$
}

\section{RESUMEN}

Este artículo tiene como objetivo caracterizar la informalidad laboral de los vendedores ambulantes y estacionarios ubicados en el espacio público del centro de Villavicencio (Colombia) durante el año 2016. Para esto, se hizo un levantamiento cartográfico de los emplazamientos donde se ubican los vendedores informales y un muestreo del ruido por decibeles mediante la aplicación Decibel 10 ht ${ }^{\circledR}$; además, se identificó el perfil de los vendedores por medio de una observación participativa y entrevistas semiestructuradas. Los resultados evidencian un proceso de clusterización espacial, determinado por la localización en vías peatonales, intersecciones y accesos a lugares concurridos. Se observó especialización por productos, asociada a la estructura del comercio formal.

\section{PALABRAS CLAVE}

Economía informal; análisis regional y urbano; comercio minorista; vendedores ambulantes; informalidad laboral; Colombia

\section{CLASIFICACIÓN JEL}

E26, J46, O18, L81

\section{CONTENIDO}

Introducción; 1. Estudios sobre informalidad laboral en el espacio público; 2. Diseño metodológico; 3. Resultados; 4. Conclusiones; Bibliografía.

* Este artículo de investigación es producto del proyecto de investigación: "Caracterización del mercado de trabajo en Colombia. Un análisis desde la perspectiva de la segmentación laboral", adscrito al Grupo de Investigación Economía Internacional (Clasificado en Categoría Al por Colciencias en 2017), financiado por la Universidad de Manizales y ejecutado durante los años 2015 y 2016.

** Economista, Universidad de Manizales (Manizales, Colombia). Magíster en economía, Universidad de Manizales (Manizales, Colombia). Integrante del Grupo de Investigación Economía Internacional, Universidad de Manizales. Investigador, Observatorio del Mercado de Trabajo de Caldas. Profesor de tiempo completo, Facultad de Ciencias Contables, Económicas y Administrativas, Universidad de Manizales. Correo electrónico: ecastro@umanizales.edu.co.

".. Economista, Universidad de Manizales (Manizales, Colombia). Magíster en gerencia del talento humano, Universidad de Manizales (Manizales, Colombia). Doctor en administración, Universidad Andina Simón Bolívar (Quito, Ecuador). Decano, Facultad de Ciencias Contables, Económicas y Administrativas, Universidad de Manizales. Integrante del Grupo de Investigación Economía Internacional, Universidad de Manizales. Correo electrónico: merca2@umanizales.edu.co.

${ }^{* * * *}$ Administrador de Empresas, Universidad Nacional de Colombia (Manizales, Colombia). Magíster en Economía, Universidad de Manizales (Manizales, Colombia). Líder del Grupo de Investigación Economía Internacional, Universidad de Manizales. Investigador del Observatorio del Mercado de Trabajo de Caldas y profesor de tiempo completo, Facultad de Ciencias Contables, Económicas y Administrativas, Universidad de Manizales. Correo electrónico: hserna@umanizales.edu.co. 


\title{
INFORMAL SALES IN THE PUBLIC SPACE IN VILLAVICENCIO (COLOMBIA)
}

\begin{abstract}
This paper aims to characterize the labor informality of street vendors and stable vendors located in the public space of the downtown area of Villavicencio (Colombia) during 2016. In order to achieve this, a cartographic survey of the locations where the informal vendors are located and a decibel noise sampling using the Decibel 10ht ${ }^{\circledR}$ application were carried out. In addition, the vendors' profile was identified through participatory observation and semi-structured interviews. The results show a process of spatial clustering, determined by location in pedestrian ways, intersections and accesses to crowded places. Product specialization was observed, associated with the structure of formal trade.
\end{abstract}

\section{KEYWORDS}

Informal economy; regional and urban analysis; retail trade; street vendors; labor informality; Colombia.

\section{JEL CLASSIFICATION}

E26, J46, O18, L81

\section{CONTENT}

Introduction; 1. Studies on Labor Informality in the Public Space; 2. Methodological Design;

3. Results; 4. Conclusions; Bibliography.

\section{VENDAS INFORMAIS NO ESPAÇO PÚBLICO EM VILLAVICENCIO (COLÔMBIA) RESUMO}

Este artigo tem como objetivo caracterizar a informalidade laboral dos vendedores ambulantes e estacionários localizados no espaço público do centro de Villavicencio (Colômbia) durante o ano de 2016. Para tanto, realizou-se um levantamento cartográfico dos lugares onde os vendedores informais se posicionam e uma amostragem de ruídos por decibéis usando o aplicativo Decibel 10ht@; além disso, identificou-se o perfil dos vendedores por meio de uma observação participativa e entrevistas semiestruturadas. Os resultados evidenciam um processo de clusterização espacial, determinado pela localização em vias para pedestres, intersecções e acessos a lugares concorridos. Observou-se especialização por produtos, associada à estrutura do comércio formal.

\section{PALAVRAS-CHAVE}

Economia informal; análise regional e urbana; comércio varejista; vendedores ambulantes; informalidade laboral; Colômbia.

\section{CLASSIFICAÇÃO JEL} E26, J46, O18, L81

\section{CONTEÚDO}

Introdução; 1. Estudos sobre informalidade laboral no espaço público; 2. Design metodológico; 3. Resultados; 4. Conclusões; Bibliografia. 


\section{INTRODUCCIÓN}

La informalidad laboral que se produce en el espacio público es un fenómeno característico de ciudades ubicadas en países con una estructura económica de corte preindustrial, en las que se puede apreciar una marcada tercerización de la estructura productiva y una clara vocación hacia el comercio no especializado en el que la base de la economía está poco diversificada. El fenómeno surge en el marco de la fragilidad normativa del Estado y una estructura fiscal agobiante para el empresario. Asimismo, se hace presente en espacios donde hay una fuerte tendencia hacia las modalidades de trabajo por cuenta propia, donde el crecimiento migratorio es exponencial y las brechas estructurales en la distribución del ingreso se manifiestan en desequilibrios como el acceso al mercado laboral, la cobertura en seguridad social y el sistema educativo. Este ha sido un tema de interés en el ámbito mundial por las singularidades que hacen del fenómeno un foco de ilegalidad, inseguridad y competencia desleal, que afecta las condiciones para el desarrollo de la vida empresarial, la movilidad vehicular y peatonal, y que contribuye de manera significativa al deterioro urbano.

En el caso colombiano, según fuentes de entidades oficiales como el Departamento Administrativo Nacional de Estadística (DANE), para el trimestre móvil de noviembre de 2017 y enero de 2018, el trabajo informal en las 23 principales ciudades alcanzaba alrededor del $48,1 \%$ del total de vinculados al mercado laboral (DANE, 2018a). Esto en un escenario donde se viene presentando una disminución del desempleo, pero la informalidad está aumentando y la calidad del empleo se está reduciendo, en especial en el sector de comercio y servicios.

Para la ciudad de Villavicencio se observa un panorama laboral en el que la mayor ocupación se produce en los sectores de comercio, restaurantes y hoteles; servicios comunales, sociales y personales; y en las actividades de transporte, almacenamiento y comunicaciones, entre otros. Durante 2016, en el departamento del Meta el sector secundario representó apenas el 9,22\% del producto interno bruto (PIB) -la industria manufacturera aporta el 1,9\% del PIB-; el comercio, el 5,6\%; y los servicios, el 17,9\%. Las demás actividades productivas de la región son extractivas (petróleo) y de agricultura (cuentas departamentales - DANE). Este panorama, y la fragilidad que produce la concentración del empleo en el sector primario, se agrava con la desaceleración económica que se presentó durante el período 2015-2016 en las tasas de crecimiento, especialmente en los sectores de construcción (-20,2\%), industria manufacturera $(-15,7 \%)$ y explotación de minas y canteras $(-14,2 \%)$ (DANE, 2018b). Bajo esas condiciones, según datos de la Gran Encuesta Integrada de Hogares (GEIH) del DANE, en la ciudad de Villavicencio la tasa de desempleo en el primer trimestre de 2017 fue del 13,9\%; la de informalidad, del 56,5\%; y la de trabajo por 
cuenta propia, del 40,6\%. Todas estas condiciones contribuyen a incentivar el trabajo informal y promover iniciativas de economías de "rebusque".

Asimismo, el elevado crecimiento migratorio hacia las zonas urbanas agrava este fenómeno ya que se estima que la migración a la ciudad de Villavicencio es alta, de acuerdo con la diferencia entre el crecimiento vegetativo y migratorio de la población registrada en las estadísticas oficiales del DANE. Su población se caracteriza por ser oriunda de la región central y oriental del país (70,0\% de los inmigrantes provienen del Meta y Cundinamarca), y de municipios como Acacías, Granada, Vista Hermosa, Puerto López, San José del Guaviare y Yopal.

De acuerdo con el trabajo de observación realizado en campo, se estima que hay más de 300 vendedores informales en el espacio público del centro de la ciudad de Villavicencio, aglomerados en las intersecciones, en las zonas más concurridas por transeúntes y en lugares donde las autoridades han sido permisivas. Además, predomina la venta de comidas, productos perecederos, ropa y otros elementos exhibidos en puestos semiestacionarios. Estas actividades pueden tener una asociación con los problemas de inseguridad y movilidad en esta zona. Según datos de la Policía Nacional, registrados en enero de 2016 y publicados por el diario El Tiempo (2016), hubo un aumento de los actos delincuenciales respecto al mismo período del año anterior, por lo cual la percepción de inseguridad ha incrementado en las calles; y es frecuente que los delincuentes pasen desapercibidos entre este tipo de actividades, o bien trabajen en redes informales para la comercialización de productos hurtados y para el microtráfico de drogas.

Según lo observado en el proceso de investigación, la movilidad también constituye un problema, ya que un porcentaje importante de la infraestructura diseñada para la movilidad peatonal y vehicular ha sido ocupado por los emplazamientos informales sobre los andenes, las bahías de estacionamiento y las esquinas de las calles. El ruido también es un problema por cuenta del perifoneo y el voceo en la calle: sumado al ruido vehicular del centro puede alcanzar niveles de más de 75 decibeles en algunas zonas, según el ejercicio de muestreo que se hizo en esta investigación. De esta manera, los puntos de mayor ruido están asociados a un alto grado de aglomeración de vendedores informales en el espacio público.

Desde las instituciones nacionales y locales se reconocen experiencias para tratar el problema de las ventas informales en el espacio público, que apuntan a procesos de reubicación, capacitación y control, así como al fortalecimiento institucional, mediante mesas sectoriales; no obstante, este problema se cataloga en los estudios académicos como una falla del Estado por la permisibilidad y la falta 
de continuidad en los procesos de intervención, mitigación y control. En la ciudad de Villavicencio se han promovido algunas iniciativas de formalización del trabajo en el espacio público, a la vez que se ha promovido la reubicación para recuperar la zona del Alto Barzal, la Avenida 40 y las plazoletas de los Centauros y la Cruz; sin embargo, las medidas no han sido efectivas, dado que el problema ha ganado fuerza en otras zonas claves del centro de la ciudad.

A partir del contexto y las evidencias teóricas se identificaron 18 problemáticas; por consenso con un grupo conformado por docentes y estudiantes de la Maestría en Economía, ofrecida por las universidades de Manizales y de los Llanos, estas se depuraron para reconocer cuáles problemáticas son causantes y cuáles son efecto. De este modo, los problemas evaluados a partir de las relaciones de causalidad fueron los siguientes: 1), la informalidad en el espacio público es receptora de población vulnerable al desempleo (ítem 1); 2), sigue un claro patrón de localización espacial (ítem 2); 3), se asocia a los problemas de inseguridad y deterioro urbano (ítem 3); 4), se produce en el marco de las deficiencias institucionales para hacer intervención, mitigación y control (ítem 4); 5), se integra al sector informal mediante el rebusque, dándole un calificativo favorable a su trabajo por la autonomía, flexibilidad y oportunidad de gestionar ingresos para la subsistencia (ítem 5); 6), produce dificultades de movilidad peatonal y vehicular (ítem 6); y 7), se relaciona con la contaminación visual y auditiva en la zona (ítem 7). Las relaciones de causalidad se estructuraron por medio de la matriz de Vester y, a partir de esos resultados, se protocolizó un esquema lógico que garantiza una correcta secuencialidad entre preguntas, objetivos y diseño metodológico de la investigación.

En ese sentido, el trabajo buscó aportar luces frente a los siguientes cuestionamientos: ¿cuáles son las condiciones de informalidad laboral en el espacio público en el centro de la ciudad de Villavicencio (Colombia) durante el año 2016?; ¿de qué manera se localizan en la zona?; ¿cuál es el perfil de los vendedores y sus puestos de trabajo?; ¿qué percepciones tienen los vendedores acerca de su trabajo y los posibles procesos de formalización?; ¿cómo incide el trabajo informal en el espacio público sobre la movilidad peatonal?; y ¿qué efectos se advierten sobre la contaminación auditiva? Para el desarrollo integral del problema también hubiera sido conveniente conocer cuáles son las medidas que se llevan a cabo desde las instituciones locales en términos de intervención, mitigación y control de esta actividad en el espacio público; no obstante, los alcances del trabajo no permitieron hacer un sondeo institucional y de la normatividad vigente sobre el tema.

Los principales resultados del estudio permitieron reconocer patrones estructurales que son comunes en otras ciudades colombianas, tales como la organización 
geográfica, la conformación de clústeres y aglomeraciones, y la división de actividades económicas a partir de las sinergias que han logrado construir los vendedores informales en las zonas urbanas, incluso con el comercio formal y las autoridades. También se aprecia un perfil del vendedor informal en el que predominan mujeres, adultos mayores (hombres ancianos), migrantes y personas con dificultades para insertarse en el mercado laboral formal. Las perspectivas de formalización en la ciudad no han sido completamente exitosas: los procesos de mitigación y control han terminado por predisponer a los vendedores informales, al tiempo que muchos prefieren el trabajo informal por los beneficios económicos, la autonomía, la flexibilidad y la evasión tributaria que es común en esa actividad. Las externalidades que produce el comercio informal en el centro histórico de Villavicencio se advierten en mayores niveles de congestión y tráfico peatonal, y en el deterioro urbano del ruido y la percepción de desorden. No obstante, este es un efecto de la misma lógica de aglomeración económica y comercial que caracteriza los núcleos urbanos, del que incluso los vendedores formales son responsables.

Este documento se estructura en cuatro secciones. En la primera se aborda el concepto de informalidad laboral, el desarrollo sobre el espacio público y los determinantes asociados, al tiempo que se hace una revisión breve de algunos estudios realizados sobre la temática; la segunda contiene la descripción metodológica; en la tercera se presenta la sistematización de resultados; y por último se presentan las conclusiones.

\section{ESTUDIOS SOBRE INFORMALIDAD LABORAL EN EL ESPACIO PÚBLICO}

El panorama de estudios sobre informalidad laboral en el espacio público -las denominadas ventas informales- ha sido un tema estudiado en el contexto de las ciudades contemporáneas, sobre todo en los ámbitos europeo, africano y latinoamericano. La forma como surge y se organiza el comercio informal en el espacio público tiene particularidades que atraviesan de forma estructural cada espacio urbano donde tiene lugar; por ejemplo, según Van den Heuvel (2015), en las ciudades de corte preindustrial es muy común que una parte importante de la actividad económica se desarrolle en la informalidad, al margen de la regulación y las políticas de desarrollo empresarial. De hecho, podría existir algún tipo de sinergia entre los comercios formal e informal, por ejemplo, en espacios donde el mercado se ha organizado en aglomeraciones y redes por eslabones productivos. Una evidencia de ello se muestra en un estudio realizado por Hsieh y Chang (2006) para el caso de Hong Kong, donde la actividad turística y los servicios asociados a esta se articulan con el desarrollo del mercado informal aunque, como se advierte en el documento, esto trae un costo social de inseguridad muy alto. En el caso de 
Bogotá, estudiado por Rocha, Sánchez y García (2009), la presencia de las ventas callejeras transmite externalidades negativas sobre el comercio formal, la congestión peatonal, el deterioro urbanístico y la mayor percepción de inseguridad.

En este escenario, Devlin (2011), en un trabajo que hace para Nueva York, expone que la ciudad siempre estará en una constante incertidumbre entre lo que es legal e ilegal en términos de ventas ambulantes; la cuestión es que, al analizar la normatividad vigente frente las ventas informales, se pueden apreciar baches normativos ante la diversidad de características y condiciones que rodean las personas que viven de esa actividad, por lo que no se sabe hasta dónde puede limitarse el Estado sobre los derechos que tiene la población para ejercer el derecho al trabajo. De hecho, Meneses-Reyes y Caballero-Juárez (2013) muestran que en México, Argentina, Brasil, Perú y Colombia las entidades gubernamentales han sido incapaces de llevar a cabo su función primaria, y por consiguiente, se han valido de diversas estrategias para erradicar o delimitar la venta callejera sin mucho éxito.

Para Loayza (2008), esto se advierte en Perú, porque la informalidad surge cuando los costos de circunscribirse al marco legal y normativo son superiores a los beneficios que ello conlleva. Según el autor, la informalidad en dicho país es producto de la combinación de malos servicios públicos y un marco normativo que agobia a las empresas formales. En este mismo contexto, Mkhize, Dube y Skinner (2013) estudiaron el problema de informalidad en Durban (Suráfrica) mediante una revisión del impacto de las políticas y la respuesta en el tiempo por parte de los vendedores informales, para lo cual evidencian el modo en que cada cambio de gobierno imprime un sentido distinto que termina por predisponer los vendedores a la formalidad, al punto que la interacción con el Estado se vuelve represiva mediante acosos y multas. Todas estas características tienden a ser recurrentes en los estudios sobre informalidad como un problema estructural e institucional que con dificultad se ha podido mitigar, y que conduce a la permisibilidad de un mínimo vital de ventas en el espacio público donde es posible formalizarse y organizarse de forma articulada en los equipamientos urbanos, como se advierte en muchas ciudades contemporáneas (sobre todo las que tienen vocación hacia el turismo).

Otro rasgo importante sobre el comercio informal en el espacio público es la forma como este se organiza, ya que las ventas callejeras tienden a generar clústeres geográficos marcados por el precario desarrollo de la actividad. Lyons, Brown y Msoka (2012) estudiaron la manera en que se organizaban los comerciantes informales en Tanzania: encontraron en varios casos una marcada tendencia de emplazamientos en sectores estratégicos con tráfico humano y, en reiteradas veces, estas personas deambulaban de un lugar a otro para evitar conflictos con 
las autoridades urbanas, lo que explica el carácter semiestacionario de la actividad. De todas formas, pese a que no hay una condición estructurante que determine la organización geográfica, es claro que esta tiende a formar patrones de localización que pueden estar determinados por la afluencia peatonal, los espacios donde el Estado es más permisible y, sobre todo, por la manera como se han estructurado las relaciones sociales dentro de la urbanidad, como lo muestra Monnet (1996) en un estudio comparativo en Toulouse, México D. F. y Los Ángeles.

Los vendedores informales del espacio público en el ámbito urbano tienen otros rasgos distintivos en términos demográficos y sociales. Una evidencia de ello es la predominancia de personas vulnerables a las dificultades estructurales de acceder al mercado laboral; ejemplo de ello es la población adulta, sobre todo hombres mayores y mujeres. En África, Mitullah (2003) indica que la mayoría de vendedores ambulantes son mujeres, trabajadoras por cuenta propia y, en su mayor parte, dedicadas a la venta minorista. Las mujeres son cabeza de hogar, divorciadas o viudas y con bajo nivel de escolaridad que no se rigen por una lógica convencional de planeación estratégica de ventas, con lo que su trabajo es más empírico y menos académico, como lo plantean Hiemstra, Van der Kooy y Frese (2006) en una investigación desarrollada en Vietnam.

También es frecuente encontrar dentro de esta población a grupos inmigrantes de zonas rurales o internacionales. Algunas evidencias en trabajos empíricos sobre esta condición se muestran en Vietnam, Tailandia, Turquía, varias ciudades europeas y Estados Unidos. Según Turner y Schoenberger (2012), en Vietnam muchos de los vendedores que son migrantes argumentan que se desplazaron a las ciudades a desarrollar actividades informales debido a la expansión del capital inmobiliario hacia las zonas rurales.

En Tailandia el estudio de Trupp (2015) evidencia una motivación relacionada con la necesidad de encontrar trabajo en la modalidad de empleo por cuenta propia, que se asocia a motivaciones ligadas a factores económicos, sociales y emocionales. En el panorama de los migrantes internacionales, Estrada y Hondagneu-Sotelo (2011) describen un caso en Miami: allí es muy frecuente encontrar entre los vendedores informales a población de origen latino, ya que es una forma de ganarse la vida en actividades ilegales, y más aún si se tiene la condición de indocumentado. Johnson (2006) también valida este hecho para mujeres migrantes de Bolivia, México y Perú que trabajan en la venta de alimentos.

En Colombia se han realizado estudios en las zonas urbanas. Martínez y Short (2017), por ejemplo, analizaron dos grupos de comerciantes localizados en el mercado de Santa Helena en Cali: el primero se encontraba en locales comerciales; mientras 
que el segundo estaba compuesto por vendedores ambulantes ubicados en el mercado. Entre otros asuntos, los autores identificaron que la venta informal provee oportunidades laborales a personas con bajos niveles de educación y pertenecientes a minorías étnicas; además, los vendedores ambulantes reportan un mayor nivel de ingreso que los ubicados en locales comerciales -aproximadamente un 30\% más-, aspecto que va en perjuicio de programas de reubicación y formalización para dicho contexto.

Vargas y Urinboyev (2015) realizaron un estudio etnográfico con vendedores ambulantes de la ciudad de Bogotá. Las entrevistas tenían como finalidad analizar sus experiencias frente a la normatividad y los programas de formalización, lo cual permitió identificar su deseo de recibir mayor apoyo por parte de las instituciones gubernamentales, la existencia de una posición crítica contra el sistema de protección social -que no logra cubrir sus necesidades-y el rechazo a programas de reubicación. Con respecto a esto último existe consciencia por parte de los vendedores ambulantes de ir contra la ley: exponen que los procesos de formalización se limitan a expulsarlos de las calles, sin generar programas que les permitan mejorar de manera significativa sus condiciones de vida y laborales.

Rodríguez y Calderón (2015) analizaron la distribución espacial de los vendedores ambulantes y estacionarios de la ciudad de Bucaramanga; encontraron que la estructura del comercio informal se ha aglomerado y asentado alrededor de la economía formal de la ciudad. Del mismo modo, Gaitán et al. (2013), así como Muñoz, Avendaño y Giraldo (2016), caracterizaron a los vendedores informales de la ciudad de Neiva; lograron identificar altos niveles de pobreza, bajos niveles de educación y una mayor presencia de las mujeres cabeza de hogar en esta población. A su turno, Soto, Serna y Castro (2015) analizaron las condiciones socioeconómicas de los vendedores informales ubicados en el espacio público de la Plaza de Mercado de la ciudad de Manizales; encontraron que las actividades informales han generado sinergia con el comercio informal, dado que el comerciante informal compra y vende los productos perecederos de menor calidad al comerciante formal. Soledad, Galvis y Leal (2015), por su parte, analizaron el comercio informal en el área metropolitana de Cúcuta, el cual se caracteriza en gran medida por la venta de combustible informal. Estos vendedores son, en su mayoría, jóvenes con bajos niveles de educación, lo cual dificulta el acceso a oportunidades de empleo.

Todo lo anterior puede advertir varios patrones de comportamiento y características que homogenizan el desarrollo de la actividad informal en el espacio público, entre los que se encuentra la presencia de población vulnerable al desempleo, migrantes y personas con bajos niveles educativos. Asimismo, existen estándares de 
localización espacial mediados por la estructura de movilidad peatonal y vehicular, las relaciones sociales habituadas en el espacio urbano y el grado de permisibilidad del Estado, que tiende a soportar cierto grado de informalidad debido a la misma sinergia que puede producirse entre el comercio formal e informal, las restricciones de la normatividad y la defensa por el derecho al trabajo. Todas estas condiciones se suman a lo que la teoría y las evidencias empíricas advierten en términos de problemas de inseguridad, competencia desleal, deterioro urbano, dificultades de movilidad y congestión peatonal, entre otros.

\section{DISEÑO METODOLÓGICO}

El desarrollo de este estudio de caso se llevó a cabo en el centro de Villavicencio, capital del departamento del Meta (Colombia), que se localiza a 140 kilómetros de Bogotá. Para el año 2016 tenía una proyección de población de 495.227 habitantes, según las cifras oficiales del DANE (2016). La ubicación espacial de la zona de estudio correspondió al centro histórico (entre las carreras 28 y 33 y las calles 36 y 39), espacio donde se percibe mayor presencia de vendedores informales en el espacio público, y conocido como el centro fundacional de Villavicencio (véase el gráfico 1).

\section{Gráfico 1. Mapa del centro fundacional de Villavicencio.}

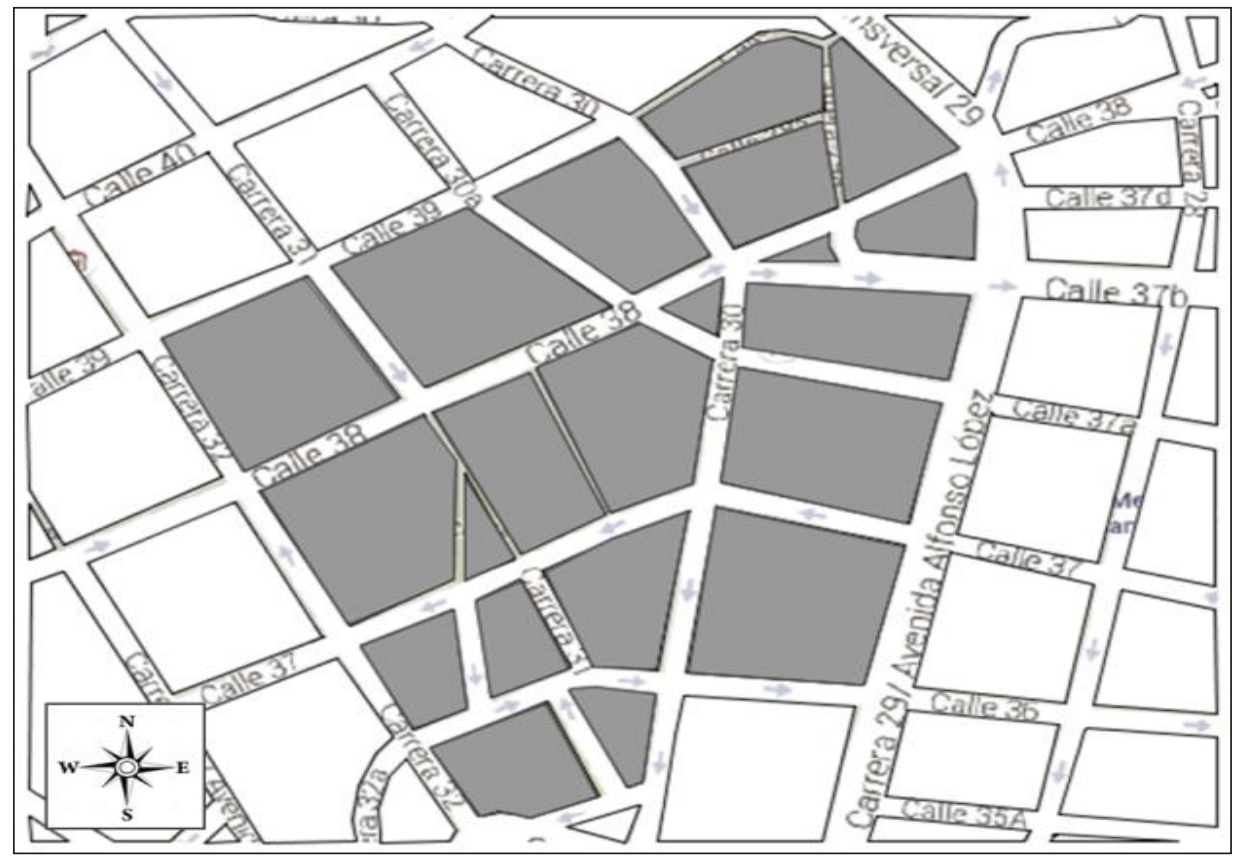

Fuente: elaboración propia. 
La matriz de Vester se estimó mediante la valoración de relaciones de causalidad entre los atributos antes identificados, y se asignó una valoración por cada uno de los 18 participantes para cada relación de causalidad entre los ítems. Cada participante otorgó una valoración de causalidad entre 0 y 4, en la que 0 es una relación que no afecta en nada y 4 es una que afecta por completo. Posterior a la valoración que dio cada participante se estimó un promedio para cada ítem, con los cuales se construyó la matriz de Vester. Luego de identificar el valor promedio que evalúa la relación de causalidad entre los problemas, se realizó una sumatoria de manera vertical (eje Y) que permite identificar los efectos; y otra de manera horizontal (eje $\mathrm{X})$ que permite reconocer las causas (tabla 1).

Tabla 1. Matriz de Vester. Esquema lógico del problema de informalidad en el espacio público de Villavicencio

\begin{tabular}{|c|c|c|c|c|c|c|c|c|}
\hline & Ítem 1 & Ítem 2 & Ítem 3 & Ítem 4 & Ítem 5 & Ítem 6 & Ítem 7 & Efectos \\
\hline Ítem 1 & 0,0 & 1,5 & 1,5 & 1,2 & 0,5 & 0,8 & 0,0 & 5,5 \\
\hline Ítem 2 & 0,0 & 0,0 & 3,0 & 0,0 & 0,5 & 3,5 & 4,0 & 11,0 \\
\hline Ítem 3 & 0,0 & 0,5 & 0,0 & 0,0 & 0,0 & 0,8 & 1,8 & 3,1 \\
\hline Ítem 4 & 1,8 & 4,0 & 3,0 & 0,0 & 1,8 & 3,0 & 2,0 & 15,6 \\
\hline Ítem 5 & 0,0 & 1,8 & 2,0 & 0,0 & 0,0 & 1,5 & 1,5 & 6,8 \\
\hline Ítem 6 & 0,0 & 0,0 & 1,5 & 0,0 & 0,0 & 0,0 & 0,5 & 2,0 \\
\hline Ítem 7 & 0,0 & 0,5 & 4,0 & 0,0 & 0,0 & 0,0 & 0,0 & 4,5 \\
\hline Causas & 1,8 & 8,3 & 15,0 & 1,2 & 2,8 & 9,6 & 9,8 & \\
\hline
\end{tabular}

Fuente: elaboración propia.

A partir de la valoración de causas y efectos se realizó un diagrama de dispersión que tiene en el eje X las causas; y en el Y, los efectos (véase el gráfico 2). Además, se usa para determinar el punto de corte la siguiente fórmula:

Corte coordenadas $(X, Y)=\frac{\text { Límite superior }- \text { Límite inferior }}{2}-$ Límite inferior

Bajo estas consideraciones se logró establecer tres escenarios de acuerdo con las estructuras de causalidad del problema, así:

1) Causas: ítems 1,4 y 5

2) Problema central: ítem 2

3) Efectos: ítems 3, 6 y 7 
La estructura de los problemas identificados se asume como objetivo de esta investigación, de modo que en el ejercicio descriptivo que se sigue a continuación se muestra un panorama de la localización de los vendedores informales (ítem 2); la relación que tiene su actividad con aspectos como el deterioro urbano; y las dificultades de movilidad y la contaminación en la zona (auditiva) (ítems 3, 6 y 7). Además, se analizan algunas características y percepciones de los vendedores acerca de su actividad, las perspectivas de formalización y la valoración en el marco de algunas iniciativas de mitigación que han visto por parte de las autoridades locales (ítems 1, 4 y 5).

\section{Gráfico 2. Diagrama de dispersión Matriz de Vester}

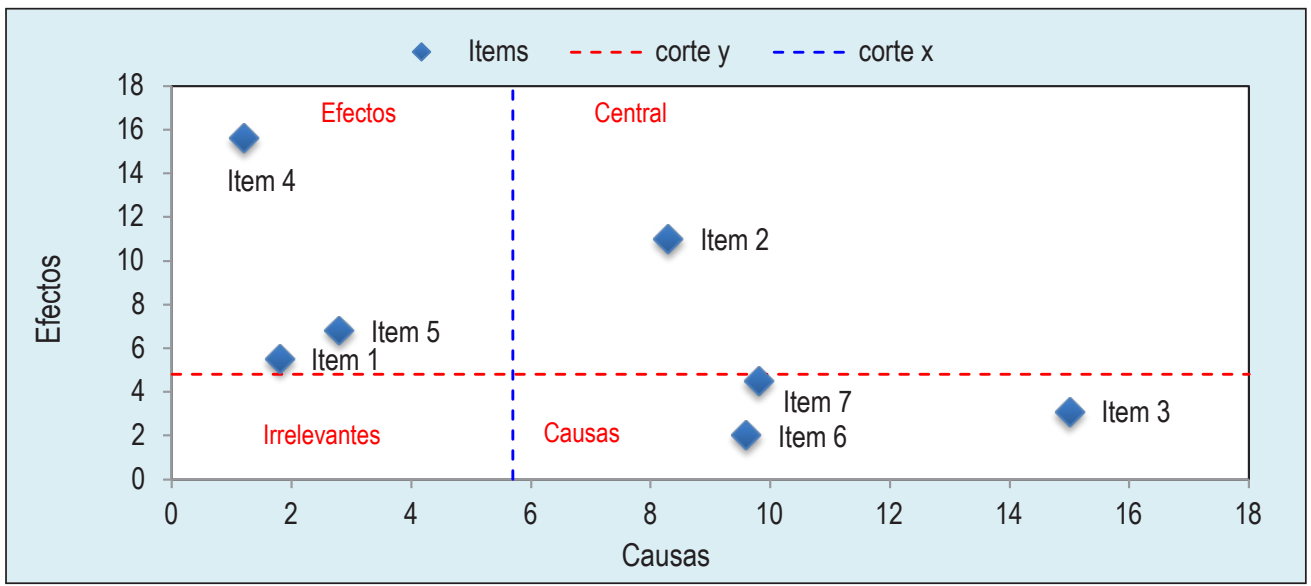

Fuente: elaboración propia.

Según el rastreo que se hizo durante el levantamiento de información cartográfica, en abril de 2016 se presentaban alrededor de 343 vendedores informales en el centro de la ciudad de Villavicencio'. Esta cifra sirvió como marco muestral para la aplicación de 61 fichas de chequeo por medio de las cuales se verificaron, mediante un proceso de observación, la localización, el género, la edad aparente, el tipo de puesto, el producto comercializado y el espacio ocupado por cada vendedor en el emplazamiento donde se ubicaban (véase el anexo 1). También se realizaron 17 entrevistas semiestructuradas en el mismo mes, con el ánimo de reconocer la percepción de los vendedores acerca de su trabajo y su posición ante posibles procesos de formalización (véase el anexo 2). Asimismo, se tomaron muestras del nivel de ruido por medio de la aplicación Decibel 10 th $^{\circledast}$ mediante el uso de dispositivos móviles; estos resultados se interpolaron en $\operatorname{ArcGIS}^{\circledR}$ para tener un panorama Para el levantamiento cartográfico se marcó en una grilla del plano de la zona, cada punto donde había
un vendedor informal, luego esta información se georreferenció con la aplicación GvSig ${ }^{\circledR}$. 
global del nivel de contaminación auditiva en el centro. La escala de decibeles que se usó como frecuencia de medición fue tomada de los parámetros de la Oficina Internacional del Trabajo - OIT (1999) de la siguiente manera:

1) $\quad 0-40 \mathrm{~dB}=$ Ruido de susurros, música a bajo volumen

2) $50 \mathrm{~dB}=$ Ruido de una conversación normal

3) $60 \mathrm{~dB}=$ Ruido irritante

4) $70 \mathrm{~dB}=$ Ruido que impide hablar.

5) $80 \mathrm{~dB}=$ Ruido leve perjudicial para daños de audición

6) $90 \mathrm{~dB}=$ Ruido medio perjudicial para daños de audición

7) 100 dB o más = Ruido lesivo perjudicial para daños de audición

8) $120 \mathrm{~dB}$ o más = Ruido que supera el umbral del dolor.

Por último, para revisar el impacto en las condiciones de movilidad peatonal, se hizo una construcción cartográfica del movimiento y estacionalidad peatonal en el centro, tomada mediante un recorrido por la plataforma de Google Street View en abril de 2016; de ese modo, se marcaron los puntos donde se muestran peatones sobre los andenes o las vías según las fotografías panorámicas de la aplicación. Esta medición se discriminó por peatones en movimiento y peatones estacionados.

\section{RESULTADOS}

\subsection{Aspectos de localización y desarrollo de la actividad}

El ejercicio de cartografía permitió identificar a 343 vendedores en el perímetro del centro de la cuidad, sin incluir los vendedores ambulantes y los estacionarios que se ubican en las casetas oficiales que fueron instaladas por la Alcaldía municipal. Los datos indican que la mayor concentración de vendedores se produce sobre las vías peatonales, las intersecciones (esquinas) y los accesos a lugares concurridos por transeúntes como plazas, paraderos de transporte público y rutas de acceso a entidades que representan la institucionalidad de la ciudad (sectores público y financiero, colegios, etc.). Asimismo, se ubican en lugares donde las autoridades locales y control de espacio público permiten un mínimo de estos puestos de trabajo, con el ánimo de mitigar la sobresaturación en otros espacios como calles e intersecciones. En el siguiente plano se muestran marcados en rojo los emplazamientos donde se ubica cada vendedor en la zona. 
Gráfica 3. Localización espacial de los vendedores informales del espacio público del centro de Villavicencio

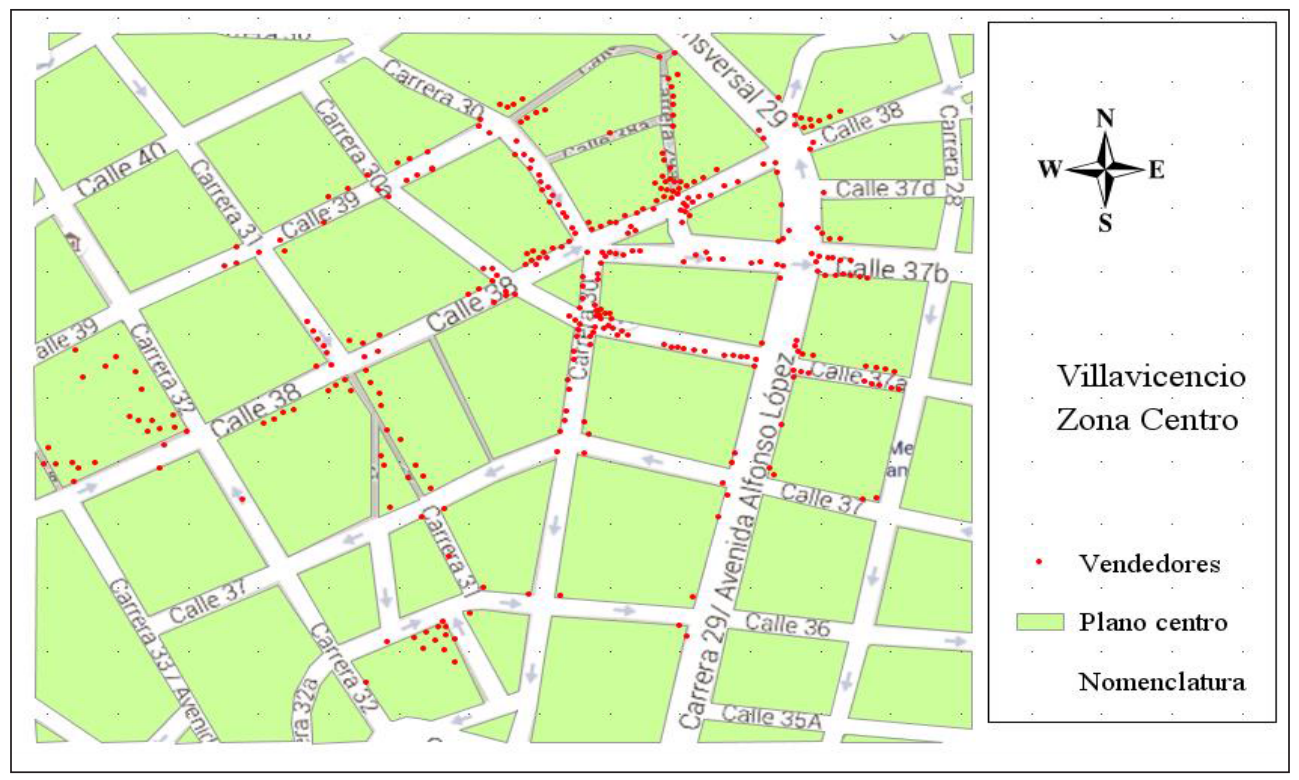

Fuente: elaboración propia.

En términos geográficos se puede notar una concentración espacial de la actividad en puntos donde los transeúntes reconocen o se han habituado a identificar cierto patrón de actividad que puede, incluso, estar asociado al comercio formal del sector; esto ocurre sobre todo con la venta de ropa y el mercado de las pulgas, la música regrabada o la comida. Este resultado corresponde a lo que Monnet (1996) describe como la estructuración de las relaciones sociales dentro de la urbanidad, lo que permite que en la construcción colectiva del paisaje urbano se reconozcan y terminen por legitimarse las actividades informales.

El chequeo en campo muestra una mayor tendencia de localización en las esquinas, en especial de vendedores de comida y productos perecederos. Además, se percibe un patrón predominante de ocupación de los andenes (52,5\% de los casos) lo que se siente al caminar por el sector, dado que en ocasiones está obstruido por completo el paso peatonal, hecho que obliga a las personas a movilizarse por las rutas de acceso vehicular. Estos resultados se pueden ver con detalle en la siguiente tabla: 
Ventas informales en el espacio público en Villavicencio (Colombia)

Tabla 2. Localización de los vendedores según la ocupación de espacio público en la zona

\begin{tabular}{|l|c|c|c|}
\hline \multicolumn{1}{|c|}{ ¿Dónde se ubica? } & 1. A media manzana & 2. En la esquina & Total \\
\hline 1. Calle & $26,7 \%$ & $54,8 \%$ & $41,0 \%$ \\
\hline 2. Andén & $63,3 \%$ & $41,9 \%$ & $52,5 \%$ \\
\hline 3. En la pared & $10,0 \%$ & $3,2 \%$ & $6,6 \%$ \\
\hline Total & $100 \%$ & $100 \%$ & $100 \%$ \\
\hline
\end{tabular}

Fuente: elaboración propia.

Un aspecto importante que determina el carácter flotante y semiestacionario de la actividad es el tipo de emplazamiento con el que se trabaja, porque además está asociado a la temporalidad y la itinerancia de esta modalidad de autoempleo. Así, se encontró que solo el 15\% de los casos chequeados en la zona tienen un emplazamiento permanente en casetas empotradas en la estructura de los andenes o las vías. Esto permite a estas personas cambiar de posición en función de la aglutinación de transeúntes en diferentes días de la semana o en momentos donde las autoridades regulan los espacios que se pueden ocupar; además, favorece el desplazamiento del puesto de trabajo en horas de la noche ${ }^{2}$, o inclusive el cambio de actividad en diferentes horarios. Por ejemplo, varios de los entrevistados indican que durante las noches o los fines de semana cambian de lugar, sobre todo los sábados y domingos, hacia lugares más concurridos que están cerca del sitio donde suelen ubicarse; de igual manera, reconocen que se presentan restricciones para la ubicación porque hay sitios dominados por otros vendedores y prefieren evitar problemas. En la tabla 3 se presentan detalles del tipo de puesto de trabajo.

Tabla 3. Tipo de puesto de trabajo de los vendedores informales

\begin{tabular}{|l|c|}
\hline \multicolumn{1}{|c|}{ Tipo de puesto } & Porcentaje \\
\hline 1. Chaza móvil & $15 \%$ \\
\hline 2. Estructura móvil (con ruedas) & $56 \%$ \\
\hline 3. Silla, trapo, mostrador & $15 \%$ \\
\hline 4. Estacionario (Caseta) & $15 \%$ \\
\hline Total & $100 \%$ \\
\hline
\end{tabular}

Fuente: elaboración propia.

2 Muchos vendedores entrevistados indican que pagan un canon de arrendamiento mensual para guardar el mobiliario de trabajo y las mercancías. 
Respecto al desarrollo de la actividad predominan las ventas de ropa y la venta de comida y perecederos. Además, hay una leve división de las actividades por género, en la que es más común la venta de comidas por parte de las mujeres y el comercio no especializado de cacharros y accesorios para electrodomésticos por parte de los hombres. Por grupos etarios solo se observa cierta tendencia en la venta de dulces y golosinas en las personas más adultas.

Otro aspecto importante encontrado en las entrevistas es la estructura de comercialización vinculante del sector formal, ya que muchos de los vendedores del espacio público indican que compran sus productos en tiendas especializadas en la misma zona y en la central de abastos. Además, algunas veces reciben suministro de energía eléctrica desde los locales del comercio formal. Esto muestra que puede haber una sinergia productiva de lo formal con lo informal como lo indican Hsieh y Chang (2006). La tabla 4 muestra la estructura de la actividad.

Tabla 4. Productos que comercializan los vendedores informales del espacio público del centro de Villavicencio

\begin{tabular}{|l|c|c|c|}
\hline \multicolumn{1}{|c|}{ Producto que vende } & Mujer & Hombre & Total \\
\hline 1. Cacharros y accesorios & $3 \%$ & $26 \%$ & $14 \%$ \\
\hline 2. Ropa y accesorios de vestir & $34 \%$ & $19 \%$ & $27 \%$ \\
\hline 3. Comida & $34 \%$ & $30 \%$ & $32 \%$ \\
\hline 4. Dulces & $16 \%$ & $19 \%$ & $17 \%$ \\
\hline 5. CD (música y películas) & $9 \%$ & $0 \%$ & $5 \%$ \\
\hline 6. Otros & $3 \%$ & $7 \%$ & $5 \%$ \\
\hline Total & $100 \%$ & $100 \%$ & $100 \%$ \\
\hline
\end{tabular}

Fuente: elaboración propia, resultados de investigación.

\subsection{Condiciones demográficas y percepciones de los vendedores sobre su actividad}

En términos demográficos se encontró una estructura ocupacional donde predominan las mujeres y las personas adultas, según lo observado en las fichas de chequeo (véase el gráfico 4). Estos resultados son comparables con las evidencias africanas desarrolladas por Mitullah (2003): en dicho trabajo se describe, en el perfil del vendedor informal, una predominancia de personas vulnerables a las dificultades estructurales de acceder al mercado laboral -en especial, las mujeres y la población adulta-. En el gráfico 4 se presenta una estructura por grupos etarios y género. 
Según comentarios de algunos de los entrevistados, durante toda su vida han desarrollado su actividad laboral en la calle y, además, se trata de una actividad de tradición familiar que vincula a otros parientes, de quienes incluso han aprendido esa labor. Estas respuestas son comparables con el caso de los vendedores informales del espacio público en la ciudad de Manizales, como lo reportan Soto, Serna y Castro (2015); se evidencia en ese caso la vinculación de estructuras familiares a la venta de productos callejeros, al tiempo que se advierte la presencia de monopolios y el usufructo de los emplazamientos que han colonizado familias completas dedicadas a esa actividad. En el centro de Villavicencio comentaba una señora: "aquí también trabaja mi mamá y mis hijos, aquí por el centro" (Anónimo, 2016, entrevista a vendedora de cinturones. Villavicencio, 16 abril de 2016).

Gráfico 4. Pirámide poblacional de los vendedores informales del espacio público del centro de Villavicencio

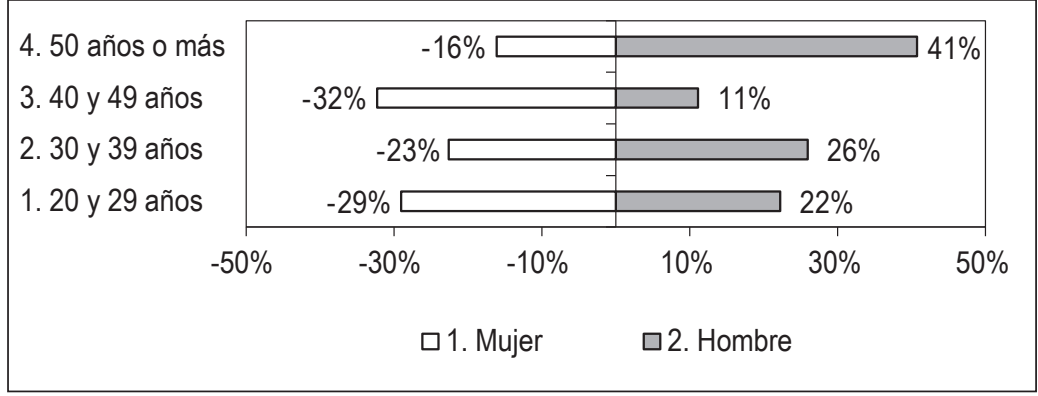

Fuente: elaboración propia.

Muchos de los entrevistados afirman que llevan una parte importante de su vida como comerciantes, apelativo que asumen para su actividad; de hecho, existe prevención sobre el calificativo "vendedores informales". Varios señalan, además, que viven en Villavicencio hace muchos años, pero llegaron a la ciudad a buscar oportunidades de empleo o como desplazados por la violencia, y luego lograron vincularse a la actividad comercial. Incluso, existen personas que trabajan de manera flotante por temporadas en otras ciudades del país, en la venta de productos en la calle. Según Trupp (2015), es común que esta actividad sea receptora de población migrante, sobre todo de zonas rurales, y esto se hace evidente para el caso de la ciudad estudiada.

La percepción generalizada sobre la actividad es favorable, en tanto es un trabajo que provee ingresos para el sustento familiar, tiene buena rentabilidad y la relación costo-beneficio es alta. Además, los entrevistados hacen alusión frecuente a la flexibilidad como algo positivo y a la autonomía que les permite administrar su horario y tomar sus propias decisiones, e inclusive trasladarse a emplazamientos 
más estratégicos para las ventas. En algunos casos, esto se percibe como un proyecto familiar para la subsistencia; y en otros, como una empresa con proyección de largo plazo. Sin embargo, se advierte sobre las dificultades de trabajar en el espacio público por la restricción que implica esa actividad y la frecuente tensión con la fuerza pública, así como por la volatilidad de los ingresos, ya que una parte importante de estas personas vive de las ventas diarias. Un testimonio de uno de los vendedores sobre la percepción de su trabajo dice lo siguiente:

Sí claro, porque por ahí se rebusca uno la plática, porque uno tiene su propio horario, a veces uno llega a las nueve a veces a las diez, (...) tampoco se puede llegar al medio día y por ahí da para los gastos, para los gastos (...) pues esto da el diario pa' los hijos (...) pues sí, eso le digo, nos toca, hay que sobrevivir ahí como pueda porque la situación esta dura y toca hacerle. (Anónimo, 2016, entrevista a vendedor de mangos. Villavicencio, 16 abril de 2016)

Muchos de los vendedores entrevistados reaccionaron de forma negativa ante un posible proceso de formalización. Entre las razones más comunes se encuentran, primero, el hecho de tener que asumir obligaciones tributarias; segundo, cumplir normas; y tercero, tener que reubicarse en otro lugar, lo que describieron como algo infructuoso, incluso ante otras experiencias de personas que se han acogido a esas iniciativas y se han visto obligadas a regresar al lugar donde trabajaban antes porque no resultó rentable la ubicación para su negocio. De esta manera, parece darse cierta predisposición a la informalidad, aunada a los casos que han sido inútiles en ese sentido. Como lo describen Meneses-Reyes y Caballero-Juárez (2013), Devlin (2011), Loayza (2008) y Mkhize, Dube y Skinner (2013), una de las principales dificultades para abordar el problema del trabajo informal en el espacio público en las ciudades se produce en el marco de las deficiencias institucionales para hacer intervención, mitigación y control. Una experiencia comentada por uno de los vendedores sobre el caso es la siguiente:

Es que formalizarse es verraco porque lo mandan para una parte que no sirve o que no se acerquen, por lo menos a los que les han dado casetas acá en el parque les ha ido muy bien, pero hay partes que no, porque han dado casetas por lo menos para barrios por allá partes que no, no les pasa nadie y no hacen nada. (Anónimo, 2016, entrevista a vendedor de mangos. Villavicencio, 16 abril de 2016).

\subsection{Efectos en la movilidad y el ambiente}

En materia de movilidad, solo se tuvo en cuenta el desplazamiento peatonal en la zona de estudio. El muestreo realizado por la aplicación de Google Street View permitió identificar a 949 peatones sobre los andenes, las calles y las plazoletas. Se identificó, además, que alrededor del 67,8\% de estos se clasifican como peatones estacionarios, ya que sobre la fotografía panorámica no estaban caminando. Esto 
puede marcar una pauta importante no solo en términos del control y ocupación del espacio público sino en materia de planificación urbana, ya que, en la lógica convencional, los andenes se conciben únicamente para el movimiento permanente de los peatones, por lo que es posible que exista congestión o tráfico denso en algunas partes -sobre todo en las más concurridas, las más estrechas o donde predomina la ocupación informal del espacio público-.

Las fotografías muestran, además, que una parte importante del tráfico peatonal se explica por la presencia de personas ubicadas alrededor de los vendedores informales. Esto se puede verificar por medio de los puntos verdes en el cartograma que muestra la estacionalidad peatonal, en especial en las intersecciones y las plazoletas (véase el gráfico 5). Los resultados permiten correlacionar los puntos más densos con la presencia de emplazamientos del comercio informal.

Sumado a lo anterior, de esta aproximación se pueden clasificar varias tipologías de peatones según la lógica funcional de desplazamiento. Algunos, por ejemplo, son estacionarios alrededor de paraderos, semáforos, bahías y otras estructuras de movilidad; mientras otros se aglomeran en plazas, se recuestan en las paredes o sobre la vía, miran vitrinas, o se ubican alrededor de establecimientos de comercio (formal e informal) y entidades oficiales. Lo anterior es un indicador de los diferentes usos y relaciones sociales que se producen en la zona del centro de la ciudad, sobre la cual valdría la pena profundizar en futuros estudios para captar las diferentes apropiaciones sociales y territoriales del espacio público en el lugar. En la siguiente grafica se muestran en rojo los peatones en movimiento; y en verde, los estacionarios.

\section{Gráfico 5. Panorámica de ocupación peatonal en el centro de Villavicencio}

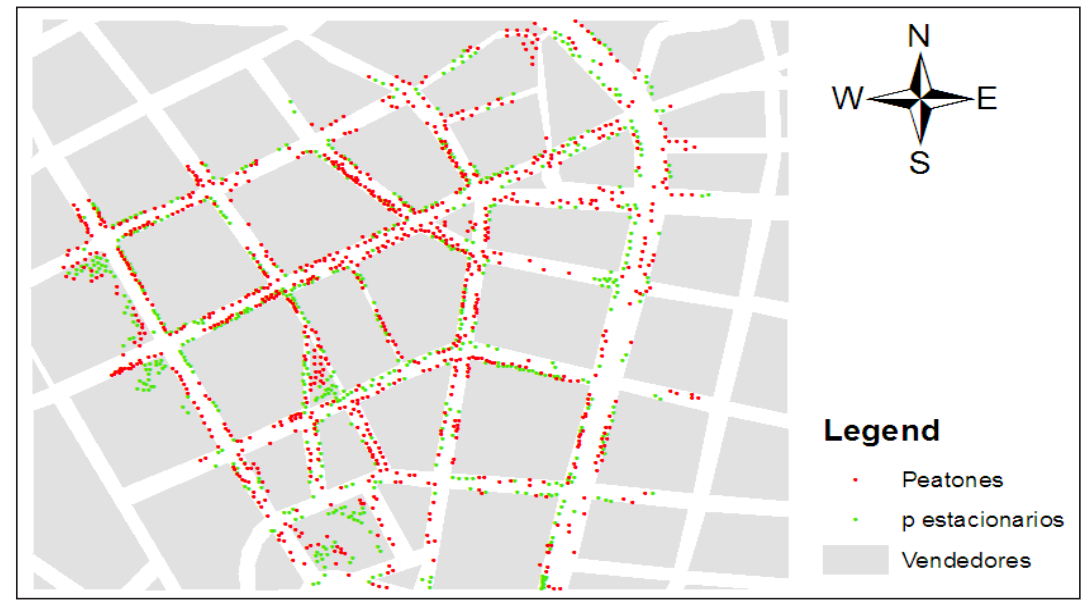

Fuente: elaboración propia con base en Google Street View. 
dispositivos móviles en 21 puntos en la zona del centro, los cuales se interpolaron para tener una panorámica del efecto de ruido. Si bien en esta zona de la ciudad los decibeles de ruido tienden a ser altos debido al tráfico vehicular, a esto se suman el perifoneo y voceo del comercio formal e informal, además del ruido de los peatones y los equipamientos para usos residenciales y comerciales. La representación cartográfica del gráfico 6 indica en cada polígono la media de los puntos cardinales en cada intersección. Esto advierte una fuerte correlación con la zona donde existe mayor concentración de vendedores informales en el centro. Durante el recorrido se pudo notar el uso de parlantes, megáfonos, baterías de amplificación (sobre todo en el comercio formal) y el voceo con el que se ofertan los productos comercializados por los vendedores. El promedio de decibeles de ruido en la zona estudiada es de 58,5, lo que se puede catalogar como una medida aceptable según la escala de la OIT para una jornada de 8 horas de trabajo. No obstante, existen lugares en áreas aledañas a la carrera 28 donde se alcanzan hasta 77 decibeles, lo que constituye un volumen que puede llegar a ser perjudicial para la salud. A este respecto, el gráfico 7 representa un plano de interpolación de Kernel sobre una grilla estimada en ArcMap $^{\circledR}$.

\section{Gráfico 6. Cartograma de decibeles de ruido}

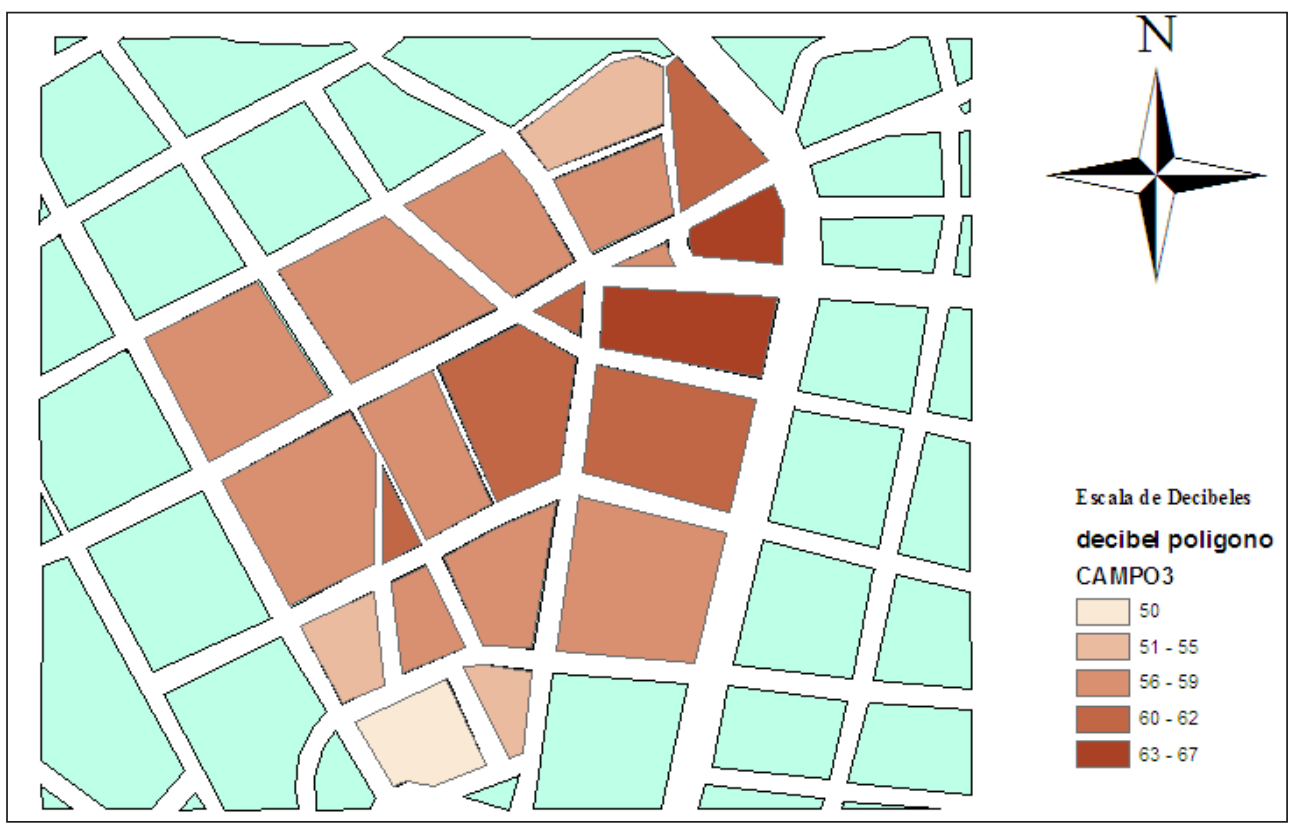

Fuente: elaboración propia. 


\section{Gráfico 7. Interpolación Kernel por decibeles de ruido}

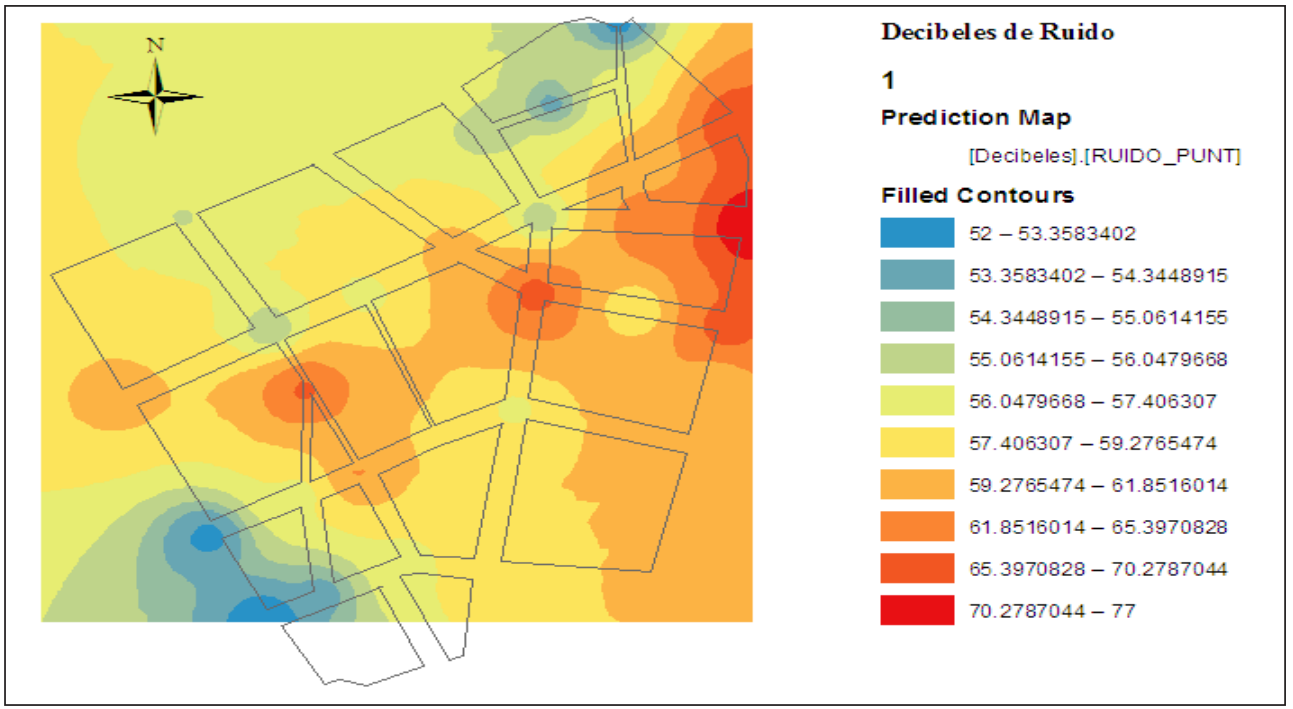

Fuente: elaboración propia.

En este panorama se puede apreciar, entonces, una concurrencia de hechos asociados a la problemática de las ventas informales en la ciudad de Villavicencio, relacionadas con el ruido; la movilidad peatonal; el tráfico vehicular; los hechos y percepciones de inseguridad; los problemas estructurales del mercado de trabajo; y las dificultades institucionales para mitigar y controlar la ocupación del espacio público. De esta manera, la intención de sistematizar este caso ha hecho posible apreciar elementos estructurantes y estructurados del fenómeno, y los efectos colaterales de la informalidad. Asimismo, se hacen evidentes algunos aspectos demostrados en varias ciudades del mundo, que se pueden catalogar como patrones característicos del vendedor informal, de las condiciones de ocupación y de los determinantes y efectos del problema. En ese sentido, se ha logrado demostrar que, para las ventas informales en el espacio público en el centro de la ciudad de Villavicencio, se manifiestan las mismas problemáticas estructurales que homogenizan esta temática en el ámbito mundial.

\section{CONCLUSIONES}

Es preciso señalar en este punto dos elementos sobre el estudio de caso realizado, uno de orden metodológico y otro de carácter analítico e interpretativo. En lo metodológico se evidencia la importancia de estudiar el problema de las ventas informales en el espacio público de manera integral desde las dimensiones eco- 
nómicas, sociales, geográficas e institucionales; esto último en lugar de hacerlo de manera fragmentada, como se ha estudiado con regularidad, bien sea desde un enfoque descriptivo por medio de caracterizaciones socioeconómicas, desde la evaluación de las iniciativas oficiales de mitigación y control, o desde los efectos sobre la movilidad, la contaminación, la inseguridad y el deterioro urbano.

Como práctica de trabajo experimental fue importante la construcción colectiva de un discurso que se operacionalizó durante el desarrollo de la investigación. Esto implicó hacer uso de herramientas esquemáticas de organización del problema mediante la matriz de Vester, la cual permitió estructurar una ruta de trabajo que fue clave en la recolección y sistematización de resultados.

Frente a lo analítico e interpretativo, y en lo que atañe a la problemática estudiada, se puede concluir que el tema de informalidad en el espacio público ostenta patrones estructurales que se replican en el espacio urbano. Entre ellos se puede advertir que, a diferencia de lo que se percibe, estos tienden a configurar esquemas de organización espacial que se aprecian en la formación de clústeres y aglomeraciones, y en la división de actividades por emplazamientos específicos mediados por las sinergias que se logra construir con el comercio formal, por la regulación de las autoridades y, especialmente, en medio de las relaciones sociales dentro del mobiliario urbano, donde los vendedores informales terminan convirtiéndose en parte del paisaje de la ciudad.

Igualmente, se notan unos rasgos distintivos sobre el perfil del vendedor informal: predominan mujeres, personas adultas, migrantes y e individuos con dificultades para insertarse en el mercado laboral formal. Además, se replican los argumentos que resultan de los procesos infructuosos de mitigación y control del problema que terminan por predisponer a los vendedores frente a las iniciativas de formalización; esto último aunado a una barrera que constituye la autopercepción favorable que tienen hacia su trabajo.

Por último, es posible validar algunas asociaciones que existen como efecto colateral del desarrollo de actividades informales en el espacio público, tales como los indicadores de congestión y tráfico peatonal, y la contaminación auditiva que se percibe en lugares circundantes a los emplazamientos informales. De todas formas, estos son hechos que además están determinados por la naturaleza misma de los centros de negocios, los centros financieros y los centros parlamentarios de las ciudades.

En este sentido, es importante que la ocupación del espacio público deba entenderse en el marco de los derechos al trabajo y a la ciudad, y en medio de la 
Ventas informales en el espacio público en Villavicencio (Colombia)

identificación e interpretación de las territorialidades y relaciones espaciales que se configuran sobre este. De igual modo, debe asumirse la labor investigativa de forma integral, de tal modo que se trate de confrontar las posiciones de todos los actores sobre el tema y, por ende, los lineamientos que produzca la divulgación científica contribuyan a generar un acercamiento oportuno entre los actores.

\section{REFERENCIAS}

Departamento Administrativo Nacional de Estadística - DANE (2016). Estimaciones de población 1985-2005 y proyecciones de población 2005-2020 nacional, departamental y municipal por sexo, grupos quinquenales de edad. Anexo estadístico de Estimaciones y proyecciones de población a junio 30 de 2016.

Departamento Administrativo Nacional de Estadística - DANE (2018a). Medición de empleo informal y seguridad social. Boletín técnico de la Gran Encuesta Integrada de Hogares (GEIH). Noviembre 2017-enero 2018, 12p.

Departamento Administrativo Nacional de Estadística - DANE (2018b). Producto Interno Bruto (PIB). Boletín técnico de las Cuentas Departamentales (2016 provisional). Febrero 15 de 2018, 14p.

Devlin, R. (2011). An area that governs itself': Informality, uncertainty and the management of street vending in New York City. En: Planning Theory, Vol. 10, n. 1, p. 53-65. DOI: 10.1177/1473095210386070

El Tiempo (2016). Ola de hurtos azota a pobladores de Villavicencio. Publicado el 5 de febrero de 2016, El tiempo.com.

Estrada, E. y Hondagneu-Sotelo, P. (2011). Intersectional dignities: Latino immigrant street vendor youth in Los Angeles. En: Journal of Contemporary Ethnography, Vol. 40, n. ${ }^{\circ}$ 1, p. 102-131. DOI: $10.1177 / 089124 \overline{161} 0387926$

Gaitán, J. W.; Pérez, V.; Ayala, J. I. y Quesada, M. (2013) Caracterización de los vendedores informales del centro de la ciudad de Neiva. Neiva: Sociedad Huilense de Economistas, 24p.

Hsieh, A. y Chang, J. (2006). Shopping and tourist night markets in Taiwan. En: Tourism Management, Vol. 27, n. ${ }^{\circ}$ 1, p. 138-145. DOI: 10.1016/j.tourman.2004.06.017

Hiemstra, A.; Van der Kooy, K. y Frese, M. (2006). Entrepreneurship in the street food sector of VietnamAssessment of psychological success and failure factors. En: Journal of Small Business Management, Vol. 44, n. ${ }^{\circ}$ 3, p. 474-481. DOI: 10.1111/j.1540-627X.2006.00183.x

Johnson, L. (2006). Hybrid and global kitchens-first and third world intersections (part 2). En: Gender, Place and Culture, Vol. 13, n. 6, p. 647-652. DOI:10.1080/09663690601019802

Loayza, N. (2008). Causas y consecuencias de la informalidad en el Perú. En: Revista Estudios Económicos, n. ${ }^{\circ}$ 15, p. 43-64.

Lyons, M.; Brown, A. y Msoka, C. (2012). (Why) Have Pro-Poor Policies Failed Africa's Working Poor? En: Journal of International Development, Vol. 24, n. 8, p. 1008-1029. DOI: 10.1002/jid.2876

Martínez, L. y Short, J. R. (2017) The informal economy of cities in the south: the case of Cali, Colombia En: International Journal of Sustainable Development and Planning, Vol. 12, n. ${ }^{\circ} 4$, p. 637-643. DOI: 10.2495/SDP-V12-N4-637-643 
Meneses-Reyes, R. y Caballero-Juárez, J. (2013). The right to work on the street: Public space and constitutional rights. En: Planning Theory, Vol. 13, n. ${ }^{\circ} 4$, p. 370-386. DOI: 10.1177/1473095213503967

Mitullah, W. (2003). Street vending in African cities: A synthesis of empirical finding from Kenya, Cote d'Ivoire, Ghana, Zimbabwe, Uganda and South Africa. Nairobi: University of Nairobi, 20p.

Mkhize, S.; Godwin, D. y Skinner, C. (2013). Informal Economy Monitoring Study: Street Vendors in Durban, South Africa. Manchester, Reino Unido: WIEGO, 56p.

Monnet, J. (1996). Espacio público, comercio y urbanidad en Francia, México y Estados Unidos. En: Alteridades, Vol. 6, n. ${ }^{\circ} 11$, p. 11-25.

Muñoz, L. A.; Avendaño, J. L. y Giraldo, J. J. (2016). Espacio público e informalidad en el microcentro de Neiva. Neiva: Ediciones de la U, 103p.

Oficina Internacional del Trabajo - OIT (1999). El ruido en el lugar de trabajo, La salud y la seguridad en el trabajo: colección de módulos, Ginebra: OIT, 26p.

Rocha, R.; Sánchez, F. y García, L. (2009). Ventas callejeras y espacio público: efectos sobre el comercio de Bogotá. En: Desarrollo y sociedad, Vol. 63, n.1, p. 245-268.

Rodríguez, G. I. y Calderón, M. I. (2015) La economía informal y el desempleo: el caso de la ciudad de Bucaramanga. En: Innovar, Vol. 25, n. 55, p. 41 -58. DOI: 10.15446/innovar.v25n55.47195

Soto, I.; Serna, H. M. y Castro, E. (2015). Caracterización socioeconómica de los vendedores informales de perecederos ubicados en el espacio público de la plaza de mercado de la ciudad de Manizales. Manizales: Centro de Publicaciones Universidad de Manizales, 68p.

Soledad, J. I.; Galvis, D. C. y Leal, R. (2015) Las condiciones socioeconómicas de los pimpineros y (as) vendedores (as) ambulantes-estacionarios en el Área Metropolitana de Cúcuta, p. 122-145. En: Ramírez, Jorge Raúl y Zambrano, Mario de Jesús (Comp.) Caracterización socioeconómica de Norte de Santander: Una aproximación desde el enfoque de capacidades humanas. Pamplona: Editorial Universidad de Pamplona, 150p.

Trupp, A. (2015). The Development of Ethnic Minority Souvenir Business over Time and Space. En: International Journal of Asia Pacific Studies, Vol. 11, p. 145-67.

Turner, S. y Schoenberger, L. (2012). Street Vendor Livelihoods and Everyday Politics in Hanoi, Vietnam The Seeds of a Diverse Economy? En: Urban Studies, Vol. 49, n. ${ }^{\circ}$ 5, p. 1027-1044. DOI: 10.1177/0042098011408934

Van den Heuvel, D. (2015). Policing peddlers: the prosecution of illegal street trade in Eighteenth-Century Dutch Towns. En: The Historical Journal, Vol. 58, n. ${ }^{\circ}$ 2, p. 367-392. DOI: 10.1017/S0018246X14000478

Vargas, Ana Maria y Urinboyev, Rustamjon (2015). Everyday forms of resistance to the law: an ethnographic study of street vendors in Bogotá, Colombia. En: Droit et Société, Vol. 3, n. 91, p. 623-638. 


\section{ANEXOS}

\section{ANEXO 1. FICHA DE CHEQUEO PARA LEVANTAMIENTO DE INFORMACIÓN}

\begin{tabular}{|l|l|l|}
\hline \multirow{2}{*}{ Ficha recolección de datos } & \multicolumn{2}{l|}{ 1. La calle } \\
\cline { 2 - 3 } & & 2. El Andén \\
\cline { 2 - 3 } & & 1. La pared \\
\hline Ubicación & 2. A media manzana \\
\hline \multirow{2}{*}{ Edad } & 3. En la esquina \\
\hline Género & & F \\
\hline Producto de vende & \multicolumn{2}{|l}{ (años) } \\
\hline \multirow{2}{*}{ Tipo de puesto } & 1. Chaza móvil \\
\cline { 2 - 3 } & & 2. Coche móvil \\
\cline { 2 - 3 } & & 3. Silla, trapo o mostrador \\
\cline { 2 - 3 } & & 4. Estacionario (caseta) \\
\hline
\end{tabular}




\section{ANEXO 2. PREGUNTAS DE ENTREVISTA SEMIESTRUCTURADA}

a. ¿¿Dónde vive actualmente? ¿Cuántos años lleva trabajando en ventas en la calle? ¿Cómo inició su actividad en esta zona de la ciudad? ¿Quién trabaja con usted?

b. ¿Por qué motivo trabaja en las ventas en el espacio público? ¿Qué apreciación tiene sobre su actividad? ¿En el futuro se ve trabajando como vendedor en la calle?

c. ¿Ha pensado en formalizarse? ¿Ha tenido conflictos con las autoridades o con otros vendedores? 